\title{
CLOUD-BASED GEOSPATIAL PLATFORM IN SUPPORT OF SUSTAINABLE DEVELOPMENT GOALS 2030: HOW TO BE PREPARED FOR EARTHQUAKE DISASTERS?
}

\author{
Saied (Adam) Pirasteh ${ }^{1 *}$, Ghazal Shamsipour ${ }^{1 *}$, Guoxiang Liu ${ }^{1}$, Rasoul Hajibagheri² ${ }^{2}$ Ali Ghasemzadeh ${ }^{2}$ Mehdi Jokar $^{2}$, \\ Habibolah Zarbakhsh², Farhad Behnamfar' ${ }^{2}$ Jonathan $\mathrm{Li}^{3}$ \\ ${ }^{1}$ Department of Surveying and Geoinformatics, Faculty of Geosciences \& Environmental Engineering, Southwest Jiaotong \\ University (SWJTU), Chengdu, China- (sapirasteh, gh.shamsipour)@swjtu.edu.cn \\ ${ }^{2}$ Institute for Disaster Management (WIDM Inc.), GeoIME Inc. Mississauga, Canada \\ ${ }^{3}$ Department of Geography and Environmental Management, University of Waterloo, Canada
}

KEYWORDS: Geospatial Information Technologies, Earthquake, SDGs, Instruction Code of Building, GeoIME, GeoRVS

\begin{abstract}
:
In July 2, 2018, the United Nations Economic and Social Council (ECOSOC) adopted a resolution of the strategic framework of disaster risk reduction. Many seismic countries have experienced challenges with natural hazards, such as earthquakes every year. Seismic safety monitoring and infrastructures, including building vulnerability assessment of earthquake are significant means to protect the safety of people and reduce the loss of property. We present cloud-based Geospatial Information Technologies in this study to support the Sustainable Development Goals (SDGs) 2030 in earthquake disaster loss reduction, mitigation, and resilience. The authors investigated and programmed the instruction building codes of the Federal Emergency Management Agency. We developed sophisticated algorithms to construct a geospatial cloud-based system to support the implementation of disaster risk reduction for strengthening infrastructures and resiliency of pre and post-earthquakes. However, the content is entirely based on the understanding of geospatial knowledge, engineering, and services to the people for a better world for future generations. The objectives of this study are to (1) participate in global sharing of experiences on utilizing geospatial information technologies to address disasters resilience and challenging issues of determining the vulnerability of buildings and estimation of risk as well as recommendation for retrofitting; and (2) developing Geospatial Infrastructure Management Ecosystem (GeoIME) including, Geospatial Rapid Visual Screening (GeoRVS) cloud-based platform. They enable the determination of the vulnerability of infrastructures, such as buildings and the estimation of risk for disaster reduction and management. This study shows that we reduced the cost and time for inspecting a building by $75 \%$ and $\% 80$, respectively. The application of this study can be used for retrofitting and rehabilitation of infrastructures like buildings and bridges for before and after earthquakes. Finally, we propose recommendations that might be helpful to countries having similar issues, and it has great potential for scalability and customization in other disasters such as floods.
\end{abstract}

\section{INTRODUCTION}

Today's challenges push everyone to ponder how we, as people living on the Earth, should play a role in SDGs to transform research into real-life practices for improved condition and a better way of living together with peace in a better world. Geospatial information technology is a collection of information communication tools (Longley et al. 2011), which uses to store, capture, manipulate, transform, analyze, and generate information related to the environment and surface of the Earth, as well as below and above the ground.

On July 2, 2018, the economic and social council at the United Nations (ECOSOC) adopted a resolution and endorsed as a guide for the Member States the strategic framework to ensure the availability and accessibility of quality geospatial information across all phases of disaster risk reduction and management. The resolution enhances the importance of the utilization of geospatial information for disaster risk reduction, such as an earthquake. In the context of SDGs, GeoIME and GeoRVS techniques for assessing infrastructures like buildings can be addressed in SDGs 11 and 13 in the aspects of disaster resilience, creating a safe and liveable city and homes with the focus on housing condition.
Earthquakes can occur almost anywhere, including Australia, Canada, England, India, Iran, Japan, Mexico, Pakistan, and the US etc. Larger earthquakes produce less frequently, the relationship being exponential. Some of the mentioned countries are exposed to many minor and major earthquakes annually. It needs significant consideration to formulate the strengthened of the infrastructures, such as buildings against future earthquakes. At present, numerous weak structures are not able to withstand a shock. Public and private developers intend to use the scientific methods to prioritize and allocate budget to reinforce the structures because of limited financial resources, time and availability of an appropriate model.

Earthquakes can destroy human infrastructure and habitat, killing and impacting large populations, especially in urban areas. Although the 2002 Bam (Pirasteh et al. 2009) Iran earthquake was considered by some to be a wake-up call, it certainly reminded others that proactive mitigation efforts pay off as damage and loss of life were minimal for such a large earthquake in a populated area. Earthquakes can affect any area within a broad zone, and it may pose a great risk to human lives and fatalities that are depending on settlement distribution and densities, in addition to building materials, engineering standards, and the like. Recently, many scientists (Barzangi 1989; Kijko and Sellevoll 1992; Berberian 1994; Berberian 1995; Zhenzhong and Lanmin 1995; Ambrasys et al. 1996;

\footnotetext{
* Corresponding author
} 
McClusky 2000; Maiti 2001; Saraf et al. 2002; Ali and Pirasteh 2004; Pirasteh et al. 2008; Alam et al. 2009; Pirasteh et al. 2009; Sadra et al. 2014; Pirasteh et al., 2015) work on challenges at the various scales by using the geospatial technologies in emergency preparedness and response. They examined the challenges that occur between human and their environment under the conditions suspected to be hazardous to life and habitat. It recommended priorities for the research, the educational, and the policy contributions insight emergency preparedness and response that to be documented.

The cost-rebuilt ratio is defined as an indicator for determining the vulnerability rate of a building. This indicator is defined for different primary sectors of a building that is computed for the whole of the building in the end. Simac et al. (1991) introduced a method concerning the short reinforced concrete buildings assessment for education purposes. In this method (i.e., screening method) the assessment of the structure takes place step by step. However, this study delivers the GeoIME cloudbased platform technology.

\section{GEOSPATIAL RAPID VISUALIZATION}

Developing platforms and services such as a geospatial rapid visual screening of building is allowing designing better building information management and enabling countries to support the implementation of SDGs. We provided a GeoIME and GeoRVS cloud-based platform that helps to the rapid visual screening process and determined the vulnerability of buildings and estimation of risk (Figure 1) and presents a recommendation for building (Figure 2). The damage index model (DIM) incorporation with geospatial information can be interactively utilized into the Geographical Information System (GIS) to compute relevant engineering parameters for analyzing data and better quality management on the proposed web and cloud. Its resilience ability allows easy accessibility of the geospatial data to evaluate buildings for earthquake mitigation and preparedness. This geospatial rapid visual screening technique is a new combination of integrated geospatial system and engineering disciplines for rapid evaluation of buildings, and for city planning by further combining GIS with building structural information, civil engineering and industrial engineering as required by the Federal Emergency Management Agency (FEMA). All this accompanied by general information of the building, visualizing the 3D model of the building, seismic data, soil data, land use data, structure, parcel, material type, foundation, ceiling, wall, floor, the interior and the exterior.

\section{METHOD}

We attempted comprehensive, sophisticated computing process to generate damage index (DI) and building score utilizing the geospatial rapid visual screening technique with the Damage Index (Figure 3) of Building (DIoB) algorithm and FEMA approach (Alam et al. 2009; FEMA 2014; Pirasteh et al. 2015). The damage index approach has been developed base on the engineering parameters like seismic data, structure, parcel and material type, foundation, ceiling, wall, floor, the interior and the exterior and further combined with geospatial and building information. Figure 4 depicts the overview flowchart of the methodology in this study.

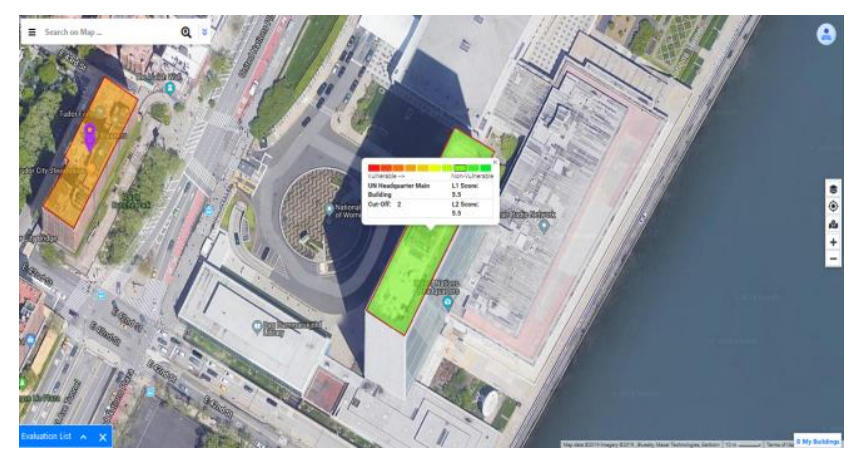

Figure 1. The vulnerability of building and estimation of risk

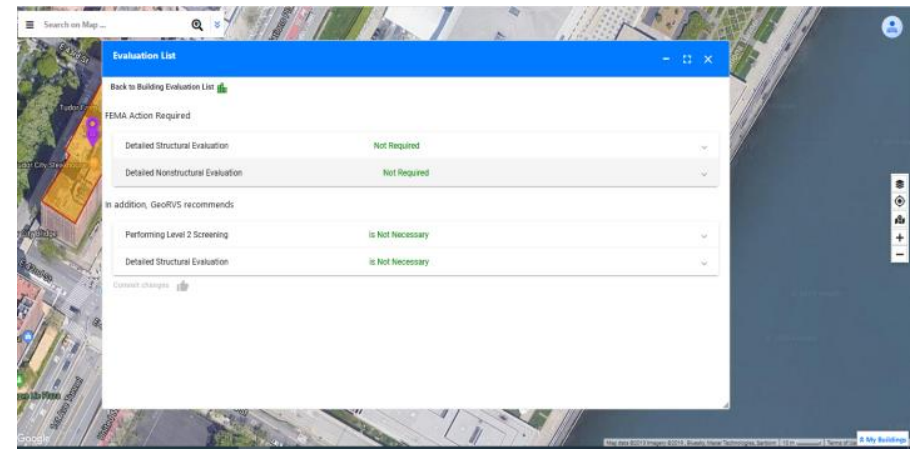

Figure 2. Recommendation for building using GeoRVS for retrofitting

The proposed algorithm (Eq. 1) was developed in this study and integrated into a geospatial cloud-based platform for data sharing and determining the vulnerability of infrastructure and estimation of risk. This algorithm developed in the MATLAB environment accompanied by the essential parameters and further adopted in Java scripts and Python to develop a geospatial infrastructure management ecosystem (GeoIME). We created Geodatabase for all data including attribute or spatial, raster or vector, integrating, analyzing, and computing the data for building evaluation on the cloud-based platform to produce maps, reports, and recommendations, and determine the prioritization of buildings for retrofitting.

This study implemented the above methods to create a smart geospatial-based platform that perhaps such an integrated geospatial platform has not been developed so far by any researchers and technical professionals in the world so far.

$$
\mathrm{DI}=\alpha_{1} \cdot \omega_{1} \sum_{\mathrm{i}=1}^{24} \beta_{\mathrm{i}}+\alpha_{2} \cdot \omega_{2} \sum_{\mathrm{i}=1}^{7} \gamma_{\mathrm{i}}+\alpha_{3} \cdot \omega_{3} \sum_{\mathrm{i}=1}^{8} \xi_{\mathrm{i}}+\alpha_{4} \cdot \omega_{4} \sum_{\mathrm{i}=1}^{5} \eta_{\mathrm{i}}+\alpha_{5} \cdot \omega_{5} \sum_{\mathrm{i}=1}^{4} \Omega_{\mathrm{i}}
$$

Where, DI is the damage index, $\alpha$ is the failure coefficient, and $\omega$ is the intensity coefficient of observed defects. $\beta$ is the effect coefficients for members, $\gamma$ is the effect coefficients for connections, $\xi$ is the effect coefficients for foundation, $\eta$ is the effect coefficients for diaphragms and $\Omega$ is the effect coefficients for interaction, so foundation acts as when larger the value of the coefficient, the larger the damage risk will found. 


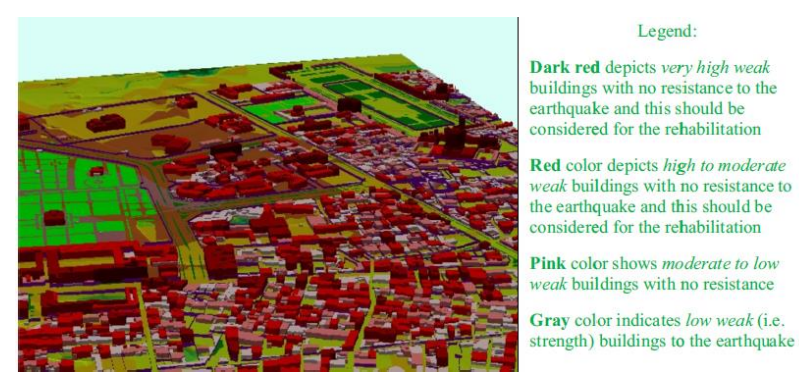

Figure 3. Classification of building damage index

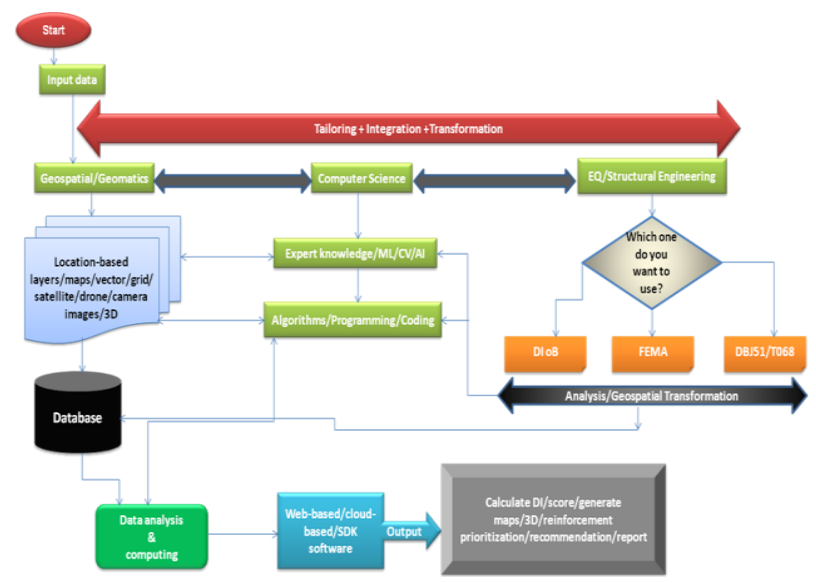

Figure 4. Flowchart of the methodology

We collected 100 samples from houses in the study area (i.e. Esfahan, Iran) as well as the first and second levels of rapid visual screening (RVS) applying FEMA-154 instruction code, from different parts of the world to examine the geospatial cloud-based platform for vulnerability assessment and estimation of risk performance. I have not used any FEMA data. Actually, FEMA-154 is the instruction for building assessment and inspection for retrofitting. We collected data from building from field observation of more than 100 buildings. We have evaluated and then compared the results of some of the samples with the FEMA existing method, which is on the paper-form. Engineering parameters such as seismic data, soil type, landslide, irregularity, structure, parcel, material type, foundation, ceiling, wall, floor, the interior and the exterior were analyzed in the field and laboratory. The output attribute data value has converted to the proposed GeoIME/GeoRVS geospatial cloud-based platform to combine with the topography city map of Esfahan, and to create maps, visualize a 3D model, generate reports. This algorithm (Eq. 1) associated with the geospatial modelling developed in this study has been used to incorporate the relationship between seismic and industrial engineering and recommend for retrofitting of the infrastructure when requires. Also, it has been considered to achieve a strategic rehabilitation management program within the proposed GeoIME/GeoRVS environment.

\section{CONCLUSION}

We developed sophisticated algorithms to create a cloud-based platform. It integrates geospatial technologies with engineering disciplines for risk assessment. It implements our proposed GeoIME/GeoRVS techniques to determine the vulnerability of infrastructures, including buildings, and estimates the risk. The proposed platform generates maps and recommendations including reports for strengthening the infrastructure such as buildings, increase mitigation of hardware and software infrastructures for disaster monitoring and warning. It also supports the disaster prevention process within the context of SDGs 2030. In this novel GeoIME integrated platform, we focus on the GeoRVS techniques that can contribute to the loss reduction and emergency response implementing SDGs 2030. GeoRVS is about resilience and introduces how to screen buildings for determining the vulnerability estimation of risk. We also present a user-friendly platform to support academicians, research and development, SDGs 2030, professionals, decision-makers, organizations, government bodies and private companies. The GeoRVS is designed to support disaster risk reduction in an actionable entrepreneurship and innovation way for a better way of implementing geospatial for a better world. The proposed GeoIME platform can be useful in building an information management system (BIMS) and applications beyond. The potential of present GeoRVS cloud-based platform shows that by adopting and customizing various data and information of a particular country, we can effectively influence the loss reduction and emergency management with the GeoIME anywhere in the world.

This study has resulted in developing GeoRVS, which is enabling the determination of the vulnerability of buildings and the estimation of risk for disaster reduction. This study shows that we reduced the cost and time for inspecting a building by $75 \%$ and $80 \%$, respectively. The application of this study can be used for retrofitting and rehabilitation of buildings. Finally, we provide recommendations to engineers and decision-makers to strengthen buildings for pre and post-earthquake disaster preparation.

Finally, we established an automated and integrated system to determine parameter correlations of different specifications. GeoIME/GeoRVS accelerates rapid visual screening for risk assessment, and screening of diversified infrastructures includes buildings, and it can be customized and localized in many countries. This platform emphasizes our contribution to the SDGs 2030 implementation plan by delivering theoretical and technical support for disaster risk reduction and resilience in countries. However, we suggest developing and integrating reconstructing 3D models and visualization by utilizing computer vision techniques for disaster risk reduction, mitigation and resilience.

\section{ACKNOWLEDGEMENTS}

The authors are thankful to the surveyors who participate in the investigation and to the screening of selected buildings. We also appreciate the Southwest Jioatong University for providing us the facilities to work in GeoAI Smarter Map and LiDAR Lab.

\section{REFERENCES}

Alam M., Mahmoodzadeh A., Pirasteh S. 2009. A Method for Rapid Evaluation of Masonry Buildings against Earthquakes, Disaster Advances Journal, 2(3): 15-23.

Ali SA., Pirasteh S. 2004. Geological application of Landsat ETM for mapping structural geology and interpretation: aided by remote sensing and GIS. Int J Remote Sens 25(21):47154727.

Ambrasys NN., Simpson KA., Bommer JJ. 1996. Prediction of horizontal response spectra in Europe, earthquake eng. Strua Dyn 25:371-400. 
Barzangi M. 1989. Continental collision zones: Sciesmotectonics and crustal structure. In: JamesDE (ed) In the encyclopedia of solid earth geophysics. Van nostrand, Rienold, New York, pp 58-75.

Berberian M.1994. Natural hazards and the first earthquake catalog of Iran. Int Inst Earthq Eng Seismol 1:266-70.

Berberian M. 1995. The first seismicity catalogue and the natural phenomenon of Iran, first edition; natural hazards prior to twentieth century, (IIEES), pp.17.

FEMA. 2014. Rapid Observation of Vulnerability and Estimation of Risk, FEMA P-154 ROVER CD Version 2, prepared by the Applied Technology Council for the Federal Emergency Management Agency, Washington, D.C.

Longley, P.A., Goodchild, M.F., Maguire, D.J. and Rhind, D.W. 2011. Geographic Information Systems \& Science (Third Edition), Wiley: Hoboken, New Jersey.

Kijko A., Sellevoll MA. 1992. Estimate of earthquake hazard parameters from incomplete data files, part ii, incorporation of magnitude heterogeneity. Bull Seismol Soc Am 82:120-134.

Maiti K. 2001. Bhuj Earthquake Mapping Surface Features Using Remote Sensing Data., M.Tech Dissertation submitted to Department of Earth Sciences. University of Roorkee, Roorkee, p 185.

McClusky. 2000. GPS constraints on plate kinematics and dynamics in the eastern Mediterranean and Caucasus. J Geophys Res 105:5695-719.

Pirasteh S., Mahmoodzadeh A., Alam M. 2008. Integration of Geo information Technology and Survey Analysis for Development in Mitigation Study against Earthquake: A Case Study for Esfahan Iran, Disaster Adv J, 1(2): 20-26.

Pirasteh S, Mahmoodzadeh A, Nikouravan B, Alam M, Asghar RSM. 2009. Probabilistic methods and study earthquakes aided by geoinformatics. Int J Geoinformatics 5(4):34-41.

Pirasteh S., Li J., Attarzadeh I. 2015. Implementation of the damage index approach to rapid evaluation building resistance for earthquake. Earth Science Informatics. 8(4):751-758. (DOI) 10.1007/s12145-014-0204-0.

Sadra K., Masakatsu M., Hassanzadeh R., Amiraslanzadeh R., Kamel B. 2014. A GIS-based seismic hazard, building vulnerability and human loss assessment for the earthquake scenario in Tabriz. Soil Dyn Earthq Eng 66:263-280.

Saraf AK., Mishra P., Mitra S., Sarma B., Mukhopadhyay DK. 2002. Remote sensing and GIS technologies for improvements in geological structures interpretation and mapping (A Technical note). Int J Remote Sens 23(13):2527-2536.

Simac MR., Bathurst RJ., Goodrun RA. 1991. Design and analysis of three reinforced soil retaining walls. Proc Geosynth 91 Atlanta GA USA 2:781-789.

Zhenzhong Z,. Lanmin W. 1995. Geological disasters in loess areas during the 1920 Haiyuan earthquake. China Geol J $36: 269-274$. 